\title{
Prevalencia del virus de Epstein-Barr en niños con leucemia del Estado de Chiapas, México
}

\section{Prevalence of Epstein-Barr virus in children with leukemia from the State of Chiapas, Mexico}

\author{
Sergio Domínguez-Arrevillaga ${ }^{1,2}$, Jesús Sepúlveda-Delgado ${ }^{1}$, Roberto A. Sánchez-González ${ }^{1,3}$, \\ Fabiola Y. Zamudio-Castellanos ${ }^{1}$, Flor E. Dávalos-Hernández ${ }^{4}$, Mónica A. Malavar-Guadarrama ${ }^{5}$ \\ y Ma. Guadalupe Trujillo-Vizuet ${ }^{1,3 *}$ \\ ${ }^{1}$ Laboratorio de Investigación, Hospital Regional de Alta Especialidad Ciudad Salud, Tapachula; ${ }^{2}$ Laboratorio de Diagnóstico Molecular, Facultad de Ciencias Químicas \\ Campus IV, Universidad Autónoma de Chiapas, Tapachula; ${ }^{3}$ Licenciatura en Médico Cirujano, Centro Universitario Cultural del Soconusco, Tapachula: ${ }^{4}$ Servicio de \\ Oncología Pediátrica, Hospital General Tapachula Dr. Manuel Velasco Suárez, Tapachula; ${ }^{5}$ Servicio de Hematología Pediátrica, Hospital de Especialidades Pediátricas, \\ Tuxtla Gutiérrez. Chiapas, México
}

\begin{abstract}
Background: The Epstein-Barr virus (EBV) is associated with the genesis of many human tumors. Of the 2 existing hypotheses regarding EBV infection, it is considered of the greatest importance to search in a timely manner for a relationship between EBV infection and the immunophenotype of acute lymphoblastic leukemia (ALL). Therefore, the objective of this study was to determine the prevalence of EBV in children with leukemia from the state of Chiapas. Methods: 62 pediatric patients with a diagnosis of leukemia were included, informed consent was obtained within the ethical recommendations. EBV was detected by real-time PCR. Results: The prevalence of EBV was $1.61 \%(n=1)(0.00 \%-4.75 \%)$ with a $95 \%$ level of confidence. Conclusions: The EBV positive case was a male patient with a diagnosis of B-ALL from the municipality of Escuintla.
\end{abstract}

Key words: Childhood leukemia. Epstein-Barr virus. Chiapas.

\section{RESUMEN}

Introducción: El virus de Epstein-Barr (VEB) está asociado con la génesis de muchos tumores humanos. De las dos hipótesis existentes con respecto a la infección por VEB, se considera de suma importancia el buscar de manera oportuna una relación entre esta infección y el inmunofenotipo de la leucemia linfoblástica aguda (LLA). Por ello, el objetivo fue determinar la prevalencia del VEB en niños con leucemia del Estado de Chiapas. Métodos: Se incluyeron 62 pacientes pediátricos con diagnóstico de leucemia; dentro de las recomendaciones éticas se obtuvo un consentimiento informado. Se realizó la detección del VEB por reacción en cadena de la polimerasa en tiempo real. Resultados: La prevalencia del VEB fue del 1.61\% $(n=1)$ (intervalo de confianza del 95\%: 0.0-4.75). Conclusiones: El caso positivo al VEB fue un paciente de sexo masculino con diagnóstico de LLA B del municipio de Escuintla.

Palabras clave: Leucemia infantil. Virus de Epstein-Barr. Chiapas.

\section{Correspondence:}

*Ma. Guadalupe Trujillo-Vizuet

E-mail: vizuet16@ hotmail.com
Date of reception: 10-06-2020

Date of acceptance: 15-04-2021

DOI: 10.24875/HMCM.20000032
Available online: $25-06-2021$

Hosp Med Clin Manag. 2020;13:149-53

2604-0018 / @ 2021 Mexican Regional Hospitals of High Specialty and Federal Hospitals. Published by Permanyer. This is an open access article under the CC BY-NC-ND license (http://creativecommons.org/licenses/by-nc-nd/4.0/). 


\section{INTRODUCCIÓN}

La etiología de la leucemia linfoblástica aguda (LLA) infantil es en gran medida desconocida, y probablemente surge de interacciones entre exposiciones exógenas y/o endógenas, además de la susceptibilidad genética ${ }^{1}$.

Existen diversas hipótesis sobre el proceso de infección del virus de Epstein-Barr (VEB) en pacientes con LLA. En algunos casos, como génesis de la enfermedad que sucede en los portadores de preleucemia, se ha informado que la ausencia de exposición a infecciones en la vida temprana y un evento genético secundario posnatal afectado por una infección tardía inducida por el estrés (segundo golpe) en el sistema inmunitario en desarrollo «no preparado» puede aumentar el riesgo de LLA infantil ${ }^{2}$.

En otros casos, las condiciones de inmunosupresión, como las complicaciones de los pacientes con cáncer, pueden conducir a la reactivación años más tarde ${ }^{3}$. Las infecciones virales son las infecciones más comunes en pacientes leucémicos pediátricos, como resultado del estado de inmunosupresión asociado a la leucemia ${ }^{4}$.

La LLA es el tipo más común de leucemia infantil y representa del 75 al $80 \%$ de la leucemia aguda (LA) en pediatría; a escala mundial y en México se ha encontrado a esta patología infantil como una con las tasas de incidencia más altas 5 .

En el Hospital de Especialidades Pediátricas, en Chiapas, se ha observado un incremento progresivo del número de casos nuevos de malignidad hematológica atendidos, hasta alcanzar la cifra de 80 casos en el año 2011. A partir de ese año se ha observado la estabilización de casos nuevos anuales alrededor de ese número, y la mayor parte de ellos (aproximadamente el 70\%) corresponden a LA .

Más del 90\% de la población se encuentra infectada con el VEB, el cual ha evolucionado sofisticadamente para sobrevivir de por vida de manera silenciosa en las células $B$ de individuos infectados. Sin embargo, si el equilibrio entre el virus y el hospedero se altera, la infección latente por VEB se podría asociar con linfomas severos.

La edad de la infección primaria por VEB varía sustancialmente a escala global y la exposición al virus al parecer se relaciona con factores socioeconómicos ${ }^{7}$. En individuos inmunocomprometidos, las células infectadas aumentan en número y finalmente las vías del control del crecimiento de las células B se activan, induciendo la transformación y llevando a las malignidades tales como carcinoma nasofaríngeo, linfoma de Burkitt, linfomas postrasplantes y carcinomas gástricos. Las malignidades asociadas al VEB tienen distribuciones geográficas distintas y ocurren particularmente con alta frecuencia en ciertos grupos sociales, indicando que los factores genéticos del hospedero pueden influir en el riesgo de la enfermedad ${ }^{8}$.

La población con leucemia infantil se encuentra inmunológicamente vulnerable a procesos infecciosos y, debido a los altos índices de población mundial con infección de VEB reportada, es de suma importancia identificar si existe infección del virus latente en la población de estudio y con ello evitar complicaciones aunadas a las que presentan por su patología. Por ello llevamos a cabo este estudio para determinar la prevalencia del VEB en niños con leucemia del estado de Chiapas.

\section{MÉTODOS}

Se realizó un estudio epidemiológico, descriptivo y transversal de pacientes pediátricos con diagnóstico de leucemia atendidos en el Hospital de Especialidades Pediátricas (HEP) y el Hospital General de Tapachula (HGT) del Estado de Chiapas. El protocolo de investigación fue aprobado por el Comité de Investigación institucional con número 015/2015. Se proporcionó a los padres de familia y/o tutores una plática sobre el motivo del estudio, así como de la importancia de su participación. Dentro de las consideraciones éticas se obtuvo un consentimiento informado con base en la Declaración de Helsinki y las pautas éticas internacionales para la investigación relacionada con la salud con seres humanos. Aceptando participar de manera voluntaria, se obtuvo una muestra de 62 pacientes con diagnóstico de leucemia con base en los criterios del Grupo Cooperativo Franco-Americano-Británico $(F A B)^{9}$ y la clasificación de la Organización Mundial de la Salud (OMS) $2008^{10}$, establecido por el Departamento de Hematología del HEP y el Departamento de Oncología Pediátrica del HGT.

Dentro de las consideraciones de bioseguridad, el manejo de residuos peligrosos biológicos infecciosos se hizo de acuerdo con lo establecido en la NOM-087ECOL-SSA1-2002. 
Se tomó una muestra de sangre por punción venosa en tubo con ácido etilenodiaminatetraacético; posteriormente se realizó el aislamiento de ADN genómico de sangre utilizando el estuche DNA Mini Kit PureLink ${ }^{\circledR}$ (Invitrogen Life Technologies, Carlsbad, CA, EE.UU.), siguiendo las instrucciones del fabricante, y los ADN se almacenaron a $-20^{\circ} \mathrm{C}$ hasta su uso. Previo a la detección del VEB, se verificó la integridad (en gel de agarosa) y calidad (amplificación de un fragmento de 262 $\mathrm{pb}$ del gen de la $\beta$-globina) del ADN.

La detección del VEB se realizó utilizando sondas $\mathrm{MGB}^{\circledR}$ y oligonucleótidos previamente reportados en la literatura9 ${ }^{9}$. Las condiciones de reacción de la qPCR (reacción en cadena de la polimerasa cuantitativa) fueron: Master Mix 2X, VEB-PROBE $25 \mu \mathrm{M}$, VEB-F $100 \mu \mathrm{M}$, VEB-R $100 \mu \mathrm{M}, \mathrm{H}_{2} \mathrm{O}$ miliQ y ADN (50 ng). Las reacciones se llevaron a cabo en el termociclador StepOne Real-Time PCR System de la marca Applied Biosystems ${ }^{\circledR}$, se utilizó AmpliRun ${ }^{\circledR}$ VEB ADN (Vircell S.L., Granada, España) como control positivo. Muestras con CT menores a 36 se consideraron positivas.

\section{ANÁLISIS ESTADÍSTICO}

Se creó una base datos en el programa estadístico IBM SPSS v. 23.0. Las variables cualitativas se representaron con frecuencias y porcentajes, y las variables cuantitativas con media y desviación estándar.

\section{RESULTADOS}

La muestra que se obtuvo fue de 62 pacientes con diagnóstico de leucemia, del HEP y HGT del Estado de Chiapas. El 50\% ( $n=31)$ fueron mujeres y el $50 \%(n=31)$ hombres, los resultados muestran una relación 1:1 en nuestra población. Con un promedio de edad fue de $10.7 \pm 4.7$ años, con una edad mínima de 4 años y una edad máxima de 18 años (Fig. 1).

Con respecto a la distribución geográfica de los pacientes con diagnóstico de LLA, se encuentran distribuidos en municipios del norte, centro y sierra del estado de Chiapas con una mayor frecuencia en Frontera con Guatemala (Fig. 2).

Se encontró una mayor frecuencia de casos de LLA $(98.4 \%)$ y solo un $1.6 \%$ de Leucemia Mieloide Crónica

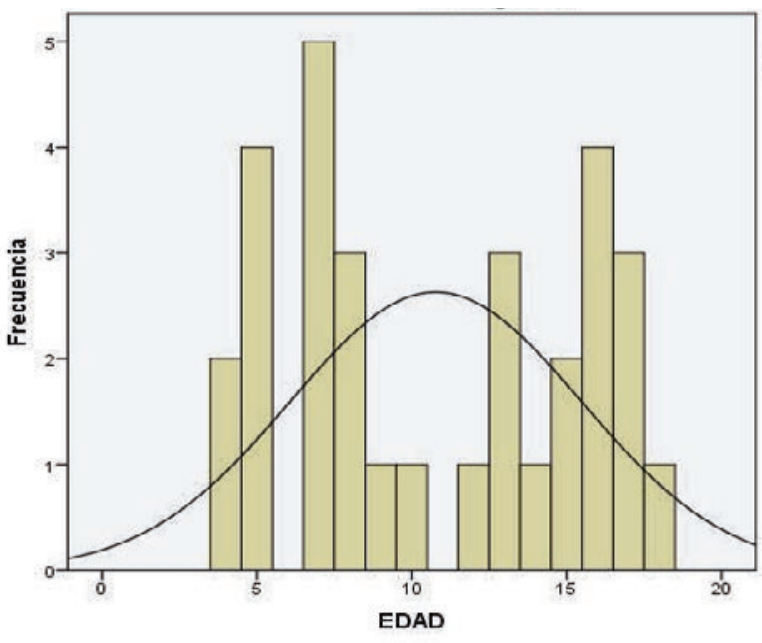

Figura 1. Histograma de la frecuencia por edades de los pacientes con diagnóstico de leucemia. (Fuente: Base de datos de participantes del HEP y HGT).

(LMC), de los cuales se reporta una prevalencia del VEB del $1.61 \%(n=1)(0.00 \%-4.75 \%)$ con un $95 \%$ de nivel de confianza (Fig. 3). El caso que presento positividad al VEB fue del sexo masculino con diagnóstico de LLA B y originario del municipio de Escuintla.

\section{DISCUSIÓN}

En México, el cáncer infantil es la primera causa de muerte por enfermedad entre los 5 y 14 años de edad. Chiapas es uno de los Estados con mayor tasa de mortalidad por cáncer en niñas y niños de 0 a 9 años y en adolescentes de 10 a 19 años; con respecto al sexo, el $56 \%$ de los casos registrados corresponde a hombres y el $44 \%$ a mujeres, de acuerdo con el Registro de Cáncer en Niños y Adolescentes (RCNA) en México en el $2019^{11 .}$

La población de estudio se encontró dentro de los parámetros reportados por el RCNA, con una edad promedio de $10.7 \pm 4.7$ años, edad mínima de 4 años y edad máxima de 18 años. La distribución porcentual por sexo se encontró un $50 \%$ de hombres y un $50 \%$ de mujeres. Con respecto a la distribución geográfica de origen, se encontró una mayor frecuencia en la región de la frontera con Guatemala. La frecuencia de casos de LLA fue mayor que la reportada por Lepe-Zúñiga, et al., debido a la participación del HGT6. El VEB puede generar el desarrollo de la leucemia o provocar un segundo golpe derivado de la inmunosupresión por la enfermedad. 


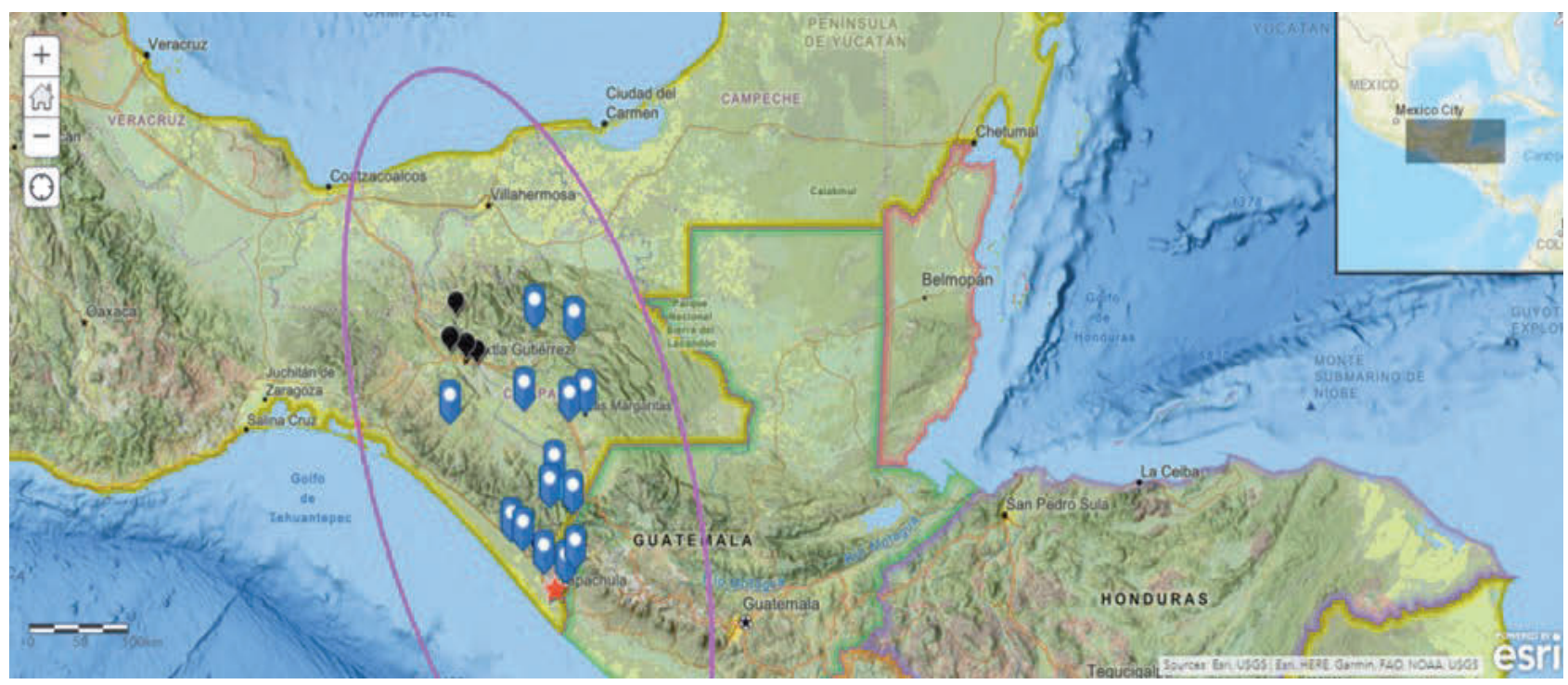

Figura 2. Localización geográfica de los pacientes con diagnóstico de leucemia en el estado de Chiapas (Fuente: ArcGis on line).

Estudios realizados en China por Guan, et al., encontraron copias de ADN del VEB en 64 de 185 (34.6\%) pacientes con LA y 2 de 37 (5.4\%) sujetos controles sanos $(p<0.001)^{8}$. La tasa de infección por VEB de pacientes con LLA, pacientes con leucemia mieloide aguda y controles sanos fue del 40.9\% (45/110), 25.3\% (19/75) y $5.4 \%(2 / 37)$, respectivamente $(p<0.05)$; los pacientes con VEB tuvieron un pronóstico desfavorable. Con base en nuestros resultados obtenidos, se considera oportuno continuar con los estudios aumentando el tamaño de muestra y utilizando controles para comparar si hay o no una diferencia significativa entre la población con LLA y los controles sanos, debido a que ellos reportan una tasa de infección del $34.6 \%$ comparando con una prevalencia del $1.62 \%$ obtenido en el Estado de Chiapas.

Loufty, et al. realizaron un estudio en Egipto para detectar la frecuencia de ADN de VEB y citomegalovirus humano (CMV) y su asociación con las características clínicas y el resultado de los pacientes con leucemia pediátrica. Las muestras de 50 pacientes leucémicos pediátricos inmunocomprometidos y 30 niños aparentemente sanos fueron sometidos a la amplificación del ADN del VEB ${ }^{12-13}$; encontraron mayor tasa de infección en LLA B. En el estudio que efectuamos el caso que fue positivo para VEB correspondió a un niño con LLA B.

La detección de la enfermedad por CMV y ADN del VEB es relativamente común en niños con leucemia y se asocia significativamente con una disminución en la supervivencia general ${ }^{14}$. EI VEB afecta a las células B; consideramos que

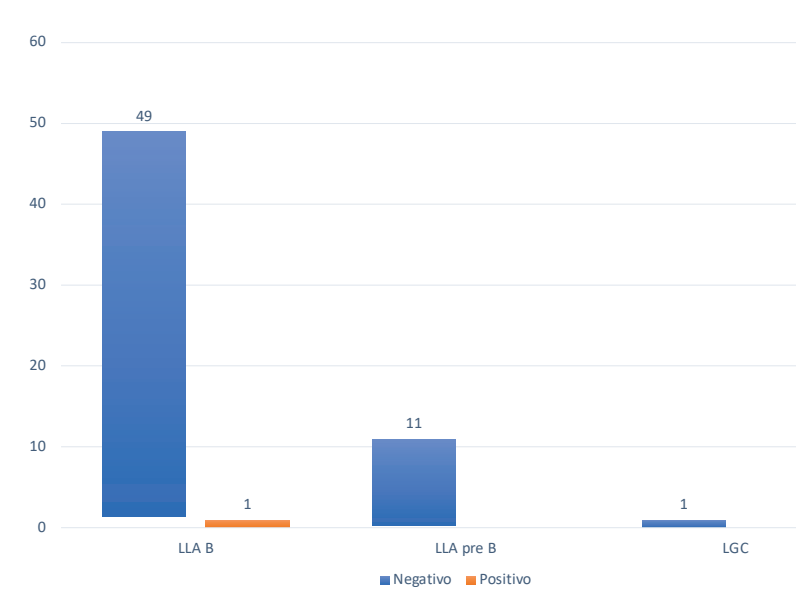

Figura 3. Frecuencia por tipos de leucemia y positividad al VEB en el estado de Chiapas (Fuente: Base de datos de participantes del HEP, HGT y resultados de la reacción en cadena de la polimerasa cuantitativala [RT-PCR]).

el resultado obtenido es consecuencia de un segundo «golpe» derivado de la inmunosupresión por la enfermedad, conduciendo a la reactivación del virus.

\section{CONCLUSIONES}

Las infecciones virales, particularmente aquellas causadas por herpesvirus, son una causa importante de morbilidad y mortalidad en pacientes inmunocomprometidos con neoplasia hematológica. Las condiciones de 
inmunosupresión, como las que enfrentan los pacientes con cáncer, pueden conducir a la reactivación años más tarde. La prevalencia del VEB en niños con leucemia del Estado de Chiapas encontrada fue del $1.61 \%$.

\section{FINANCIAMIENTO}

Proyecto Financiado por CONACyT FOSSIS: 2015-1, número 261953.

\section{CONFLICTO DE INTERESES}

Los autores no tienen ningún conflicto de intereses.

\section{RESPONSABILIDADES ÉTICAS}

Protección de personas y animales. Los autores declaran que para esta investigación no se han realizado experimentos en seres humanos ni en animales.

Confidencialidad de los datos. Los autores declaran que han seguido los protocolos de su centro de trabajo sobre la publicación de datos de pacientes.

\section{Derecho a la privacidad y consentimiento informa-}

do. Los autores han obtenido el consentimiento informado de los pacientes y/o sujetos referidos en el artículo. Este documento obra en poder del autor de correspondencia.

\section{BIBLIOGRAFÍA}

1. Greaves M. Infection, immune responses and the aetiology of childhood leukaemia. Nat Rev Cancer. 2006;6(3):193-203.

2. Hwee J, Tait C, Sung L, Kwong JC, Sutradhar R, Pole JD. A systematic review and meta-analysis of the association between childhood infections and the risk of childhood acute lymphoblastic leukaemia. Br J Cancer. 2018;118(1):127-37.

3. Cohen JI. Epstein-Barr virus infection. N Engl J Med. 2000;343(7):481-92.

4. Parasuraman R, Samarapungavan D, Venkat KK. Updated principles and clinical caveats in the management of infection in renal transplant recipients. Transplant Rev (Orlando). 2010; 24(2):43-51.

5. Global Cancer Observatory. Estimated age-standardized cancer incidence, mortality and prevalence worldwide in 2018 [Internet]. Global Cancer Observatory, International Agency for Research on Cancer, World Health Organization; 2019. Disponible en: https://gco.iarc.fr/

6. Lepe-Zúñiga JL, Jerónimo-López FJ, Hernández-Orantes JG. Características citopatológicas de la leucemia aguda en el Hospital de Especialidades Pediátricas de Chiapas, México. Bol Med Hosp Infant Mex. 2017;74(2):122-33.

7. Farrell PJ. Epstein-Barr virus. The B95-8 strain map. Methods Mol Biol. 2001;174:3-12.

8. Guan H, Miao H, Ma N, Lu W, Luo B. Correlations between Epstein-Barr virus and acute leukemia. J Med Virol. 2017;89(8):1453-60.

9. Bennett JM, Catovsky D, Daniel MT, Flandrin G, Galton DA, Gralnick HR, et al. Proposals for the classification of the acute leukaemias. French-American-British (FAB) co-operative group. Br J Haematol.1976; 33(4):451-8.

10. Sabattini E, Bacci F, Sagramoso C, Pileri SA. WHO classification of tumours of haematopoietic and lymphoid tissues in 2008: an overview. Pathologica. 2010;102(3):83-7

11. Secretaría Nacional de Vigilancia Epidemiológica/Dirección General de Epidemiología/Secretaría de Salud, México. Registro de Cáncer en Niños y Adolescentes (RCNA) en México; abril de 2019.

12. Goswami R, Gershburg S, Satorius A, Gershburg E. Protein kinase inhibitors that inhibit induction of lytic program and replication of Epstein-Barr virus. Antiviral Res. 2012:96(3):296-304.

13. Loutfy SA, Alam El-Din HM, Ibrahim MF, Hafez MM. Seroprevalence of herpes simplex virus types 1 and 2, Epstein-Barr virus, and cytomegalovirus in children with acute lymphoblastic leukemia in Egypt. Saudi Med J. 2006;27(8):1139-45

14. Loutfy SA, Abo-Shadi MA, Fawzy M, El-Wakil M, Metwally SA, Moneer MM, et al. Epstein-Barr virus and cytomegalovirus infections and their clinica relevance in Egyptian leukemic pediatric patients. Virol J. 2017;14(1):46. 